\title{
Deciphering the Complexity of Ligand-protein Recognition Pathways using Supervised Molecular Dynamics (SuMD) Simulations.
}

Alberto Cuzzolin, Mattia Sturlese, Giuseppe Deganutti, Veronica Salmaso, Davide Sabbadin, Antonella Ciancetta and Stefano Moro* .

Molecular Modeling Section (MMS), Department of Pharmaceutical and Pharmacological Sciences, University of Padova, via Marzolo 5, Padova, Italy.

*E-mail: stefano.moro@unipd.it

\section{Supporting Information Content:}

\begin{tabular}{|c|c|c|}
\hline Video Captions & Caption of Videos S1, S2, S3, S4, S5, S6. & Page S3 \\
\hline Video S1 & Acid Ellagic-CK2 recognition pathway by using SuMD. & Video-S1_2ZJW.mp4 \\
\hline Video S2 & SASP-GSTP1-1 recognition pathway by using SuMD. & Video-S2_13GS.mp4 \\
\hline Video S3 & $\begin{array}{l}\text { Benzen-1,2-diol-PDRX5 recognition pathway by using } \\
\text { SuMD. }\end{array}$ & Video-S3_4K7I.mp4 \\
\hline Video S4 & (S)-naproxen-HAS recognition pathway by using SuMD. & Video-S4_2VDB.mp4 \\
\hline Video S5 & $\begin{array}{l}\text { (S)-fluoxetine-LeuT recognition pathway by using } \\
\text { SuMD. }\end{array}$ & Video-S5_3GWW.mp4 \\
\hline Video S6 & NECA-hA $2 \mathrm{~A}$ AR recognition pathway by using SuMD. & Video-S6_2YDV.mp4 \\
\hline Video S7 & $\begin{array}{l}\text { Acid Ellagic-CK2 recognition pathway without using } \\
\text { SuMD (classical unbiased MD). }\end{array}$ & $\begin{array}{l}\text { Video-S7_non- } \\
\text { SuMD.mp4 }\end{array}$ \\
\hline Figure S1 & Examples of SuMD input (A) and $\log (\mathrm{B})$ files. & Page S3 \\
\hline
\end{tabular}




\begin{tabular}{|c|c|c|}
\hline Caption S2-12 & Caption of figures S2-S12. & Page S4 \\
\hline Figure S2 & SASP-GSTP1-1 Supplementary analysis. & Page S5 \\
\hline Figure S3 & SASP-GSTP1-1 Replicas Analysis. & Page S6 \\
\hline Figure S4 & Benzen-1,2-diol -PRDX5 Supplementary analysis. & Page S7 \\
\hline Figure S5 & Benzen-1,2-diol -PRDX5 Replicas Analysis. & Page S8 \\
\hline Figure S6 & $(S)$-naproxen-HAS Supplementary analysis. & Page S9 \\
\hline Figure S7 & (S)-naproxen-HAS Replicas Analysis. & Page S10 \\
\hline Figure S8 & $(S)$-fluoxetine-LeuT Supplementary analysis. & Page S11 \\
\hline Figure S9 & (S)-fluoxetine-LeuT Replicas Analysis. & Page S12 \\
\hline Figure S10 & NECA-hA ${ }_{2 A}$ AR Supplementary analysis. & Page S13 \\
\hline Figure S11 & NECA-hA $2 \mathrm{~A}$ AR Replicas Analysis. & Page S14 \\
\hline Figure S12 & Alternative Arg30 conformations in LeuT X-ray crystals. & Page S15 \\
\hline
\end{tabular}




\section{Caption of Videos S1, S2, S3, S4, S5, S6.}

Proteins-ligands recognition events: macromolecular systems are drown according to their secondary structure (ribbon), ligands and side chains of the amino acids mostly involved in interactions are depicted in stick. Phospholipid bilayer, if present, is shown as transparent spheres (choline polar atoms) and points (fatty tails). Intra- and intermolecular electrostatic interactions are highlighted with red dashed lines. Before to perform SuMD simulations, ligands were placed at least $30 \AA$ far from the x-ray crystallographic binding site.

\section{Caption of Videos S7.}

Classical unbiased Molecular Dynamics simulation (1 $\mu \mathrm{s})$ of the recognition pathway of CK2-Ellagic acid complex. Macromolecular system is represented according to their secondary structure (ribbon) and ligand is depicted in stick.

Figure S1. Examples of SuMD input (A) and $\log$ (B) files. In panel A, SuMD input file is presented to show the basic requirements and options needed to perform a SuMD simulation. System requirements are the structure name, the ligand 3-letter code name and a residue selection describing the binding site. SuMD parameters consist of the slope threshold and the maximum consecutive failed accepted. The simulation options include the force field, parameter file, identification number of GPU device and the randomize option. In panel B, the "SUMD.log" reports the statistics at the end of each SuMD step. In particular, the report contains the slope, the last distance registered, the interaction energies and its corresponding decomposition (electrostatic and $\mathrm{vdW}$ ). The last two columns represent the current attempt step and the termination counters, respectively.

(A)

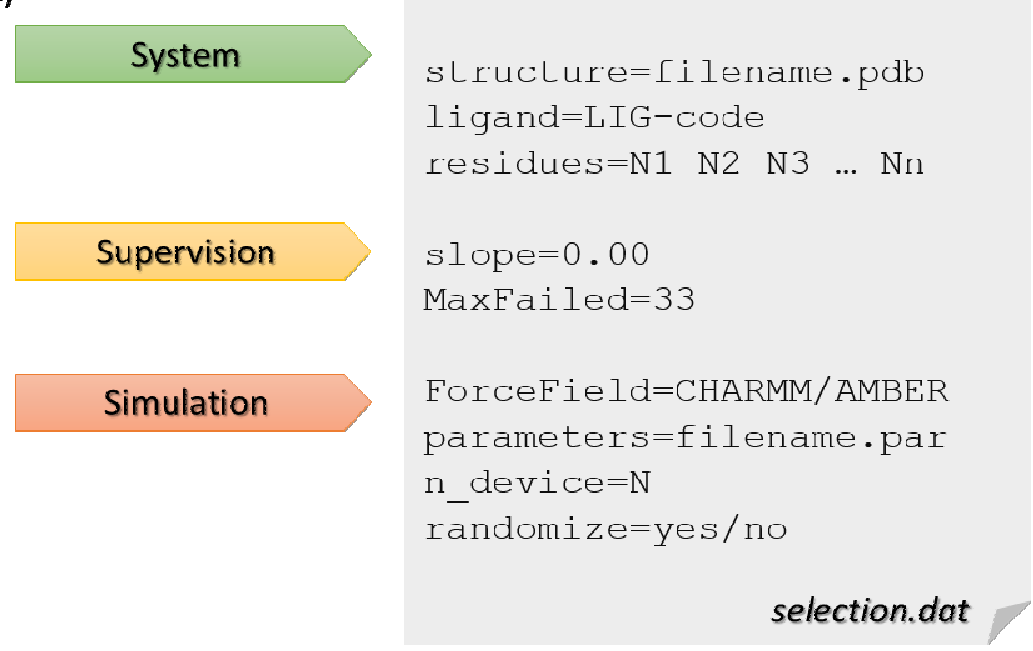

(B)

$$
\begin{aligned}
& \text { suMD_Step Slope Last_Distance Ele_Int. VdW_Int. Lig_Int. Try Dist.2 Dist.5 Dist.9 }
\end{aligned}
$$

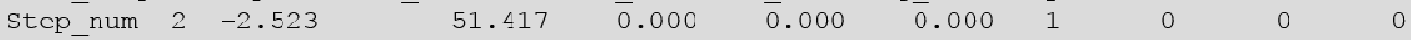

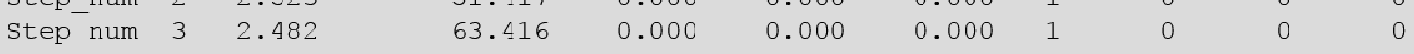

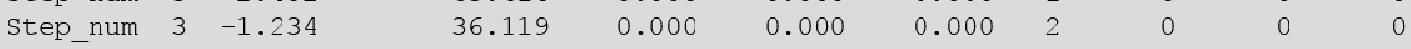

$$
\begin{aligned}
& \text {... } \\
& \text { Step_num } 16 \quad-0.889 \\
& 26.406 \quad 2.101 \quad-10.484 \quad-18.383 \quad 1 \\
& \begin{array}{lllll}
7.435 & -6.289 & -16.619 & -42.909 & 13
\end{array} \\
& 0 \quad 0 \quad 0 \\
& \text { Step_num } 30-0.282 \\
& 0 \\
& 0 \quad 19
\end{aligned}
$$




\section{Caption of Figures S2, S4, S6, S8, S10.}

Supplementary analysis: in panel A, B, C and D are reported the plots indicating the profiles of the estimated interaction energy. All of them indicate in the $\mathrm{x}$-axis the time evolution in nanoseconds of the trajectory while the $\mathrm{y}$-axis reports the estimated energy in $\mathrm{kcal} / \mathrm{mol}$. The two terms, electrostatic and van der Waals, are taken into account to estimate the Cumulative interaction and are shown in A and B. The sum of these terms, the cumulative ligand-protein interaction energy is reported in panel C. In panel D the same electrostatic (in blue) and van der Waals (in green) term and the sum of them (total, in black) are reported for each analyzed frame. In Panel D, the topology of the protein is shown using the cartoon representation and each residue is colored according to a colorimetric scale indicating the time evolution of the trajectory, from blue (first frame) to magenta (final frame), in which the first ligandresidue contact event is reported.

\section{Caption of Figures S3, S5, S7, S9, S11.}

Replicas Analysis: reported data are collected from all the SuMD simulations replicas performed.

Panel A summarizes the cumulative estimated energy interaction $(\mathrm{kcal} / \mathrm{mol})$ between the ligand and the protein, as a function of the systems time evolution (expressed in nanoseconds). In Panel B are shown the dynamic root mean square deviations (RMSD) of the ligand during the trajectories, considering the crystallographic complex conformation as reference. Panel $\mathrm{C}$ outlines the ligand-receptors recognition energy landscape $(\mathrm{kcal} / \mathrm{mol})$ : estimated energy interactions are reported as a function of the ligand distance from the binding site, and correspond to the systems evolution monitored at every SuMD checkpoint. In Panel D, the schematic ligand trajectories during all the replicas (Pollicino analysis) are reported: diameters of the spheres are proportional to the simulation time spent by the ligand at the corresponding topological protein site. 
Figure S2. SASP-GSTP1-1 Supplementary analysis.

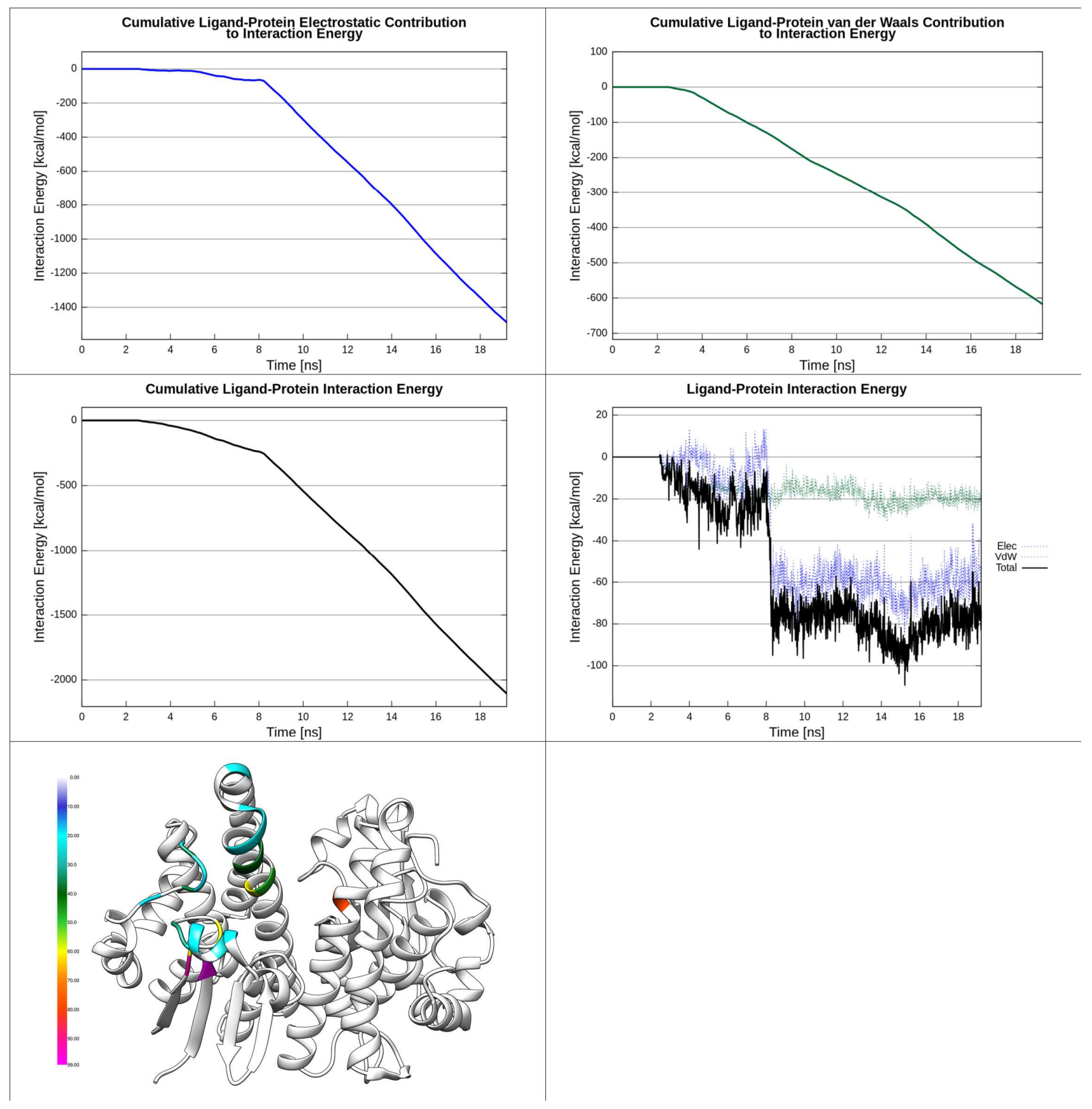


Figure S3. SASP-GSTP1-1 Replicas Analysis.
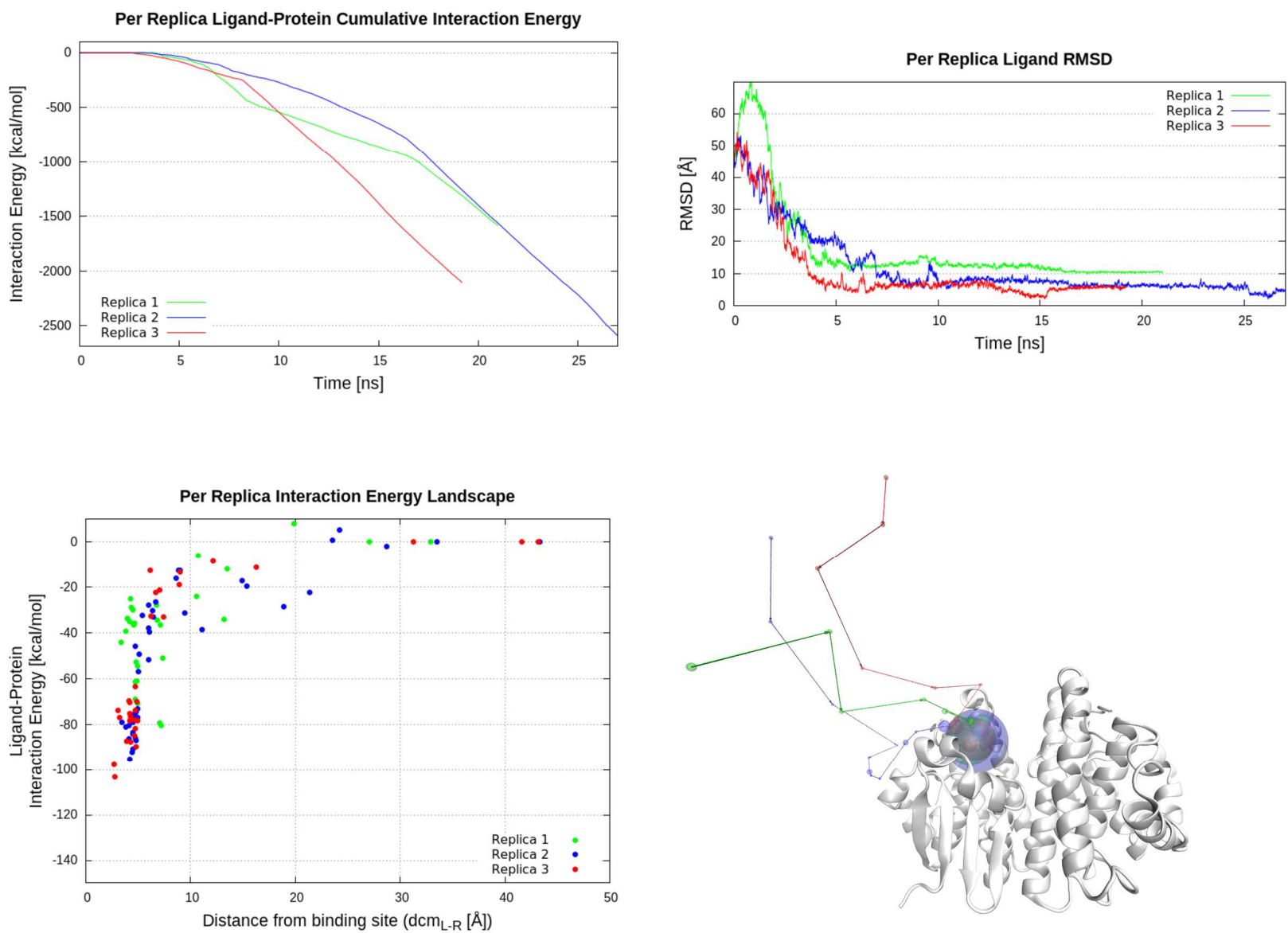
Figure S4. Benzen-1,2-diol -PRDX5 Supplementary analysis.

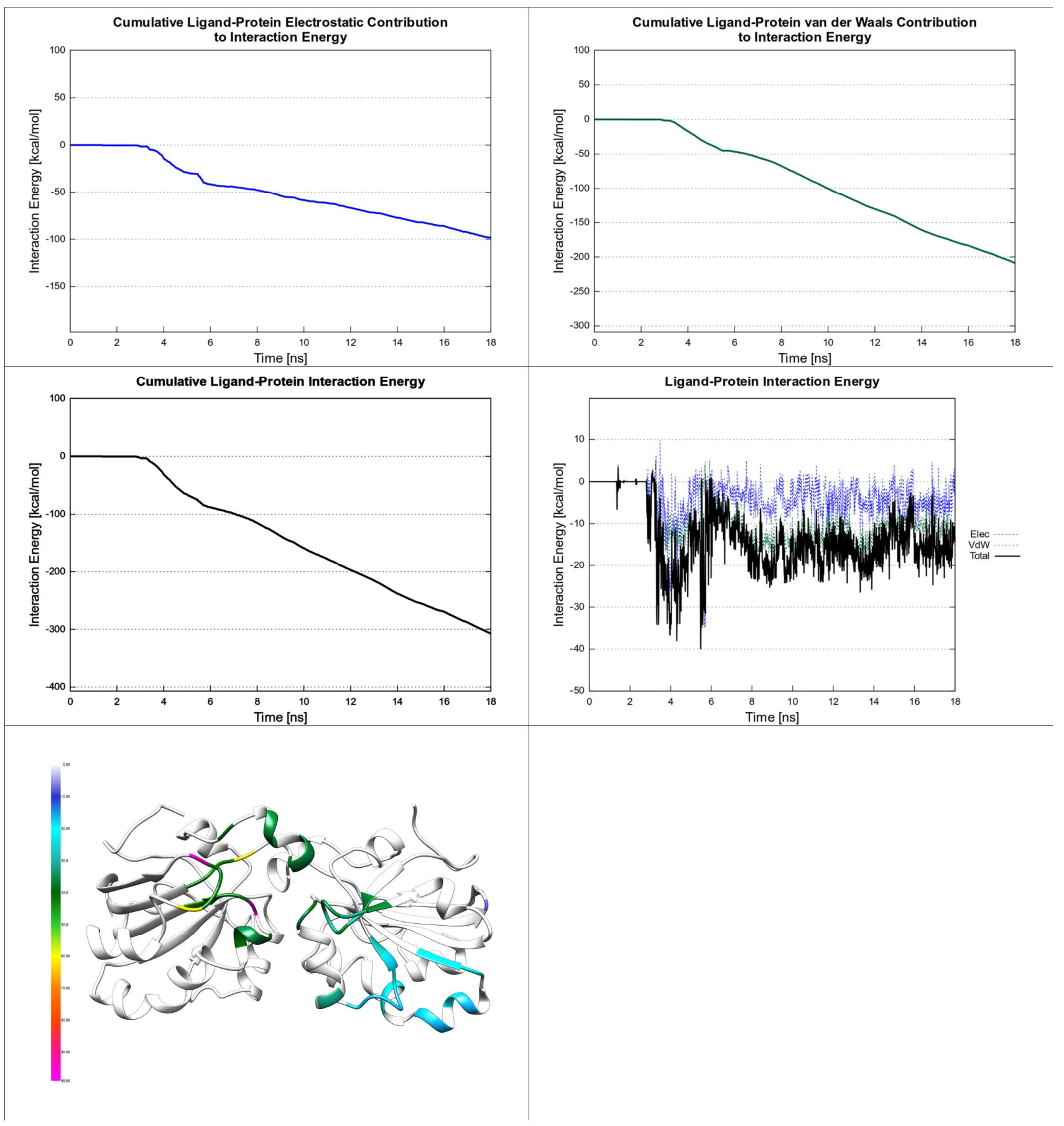


Figure S5. Benzen-1,2-diol -PRDX5 Replicas Analysis.
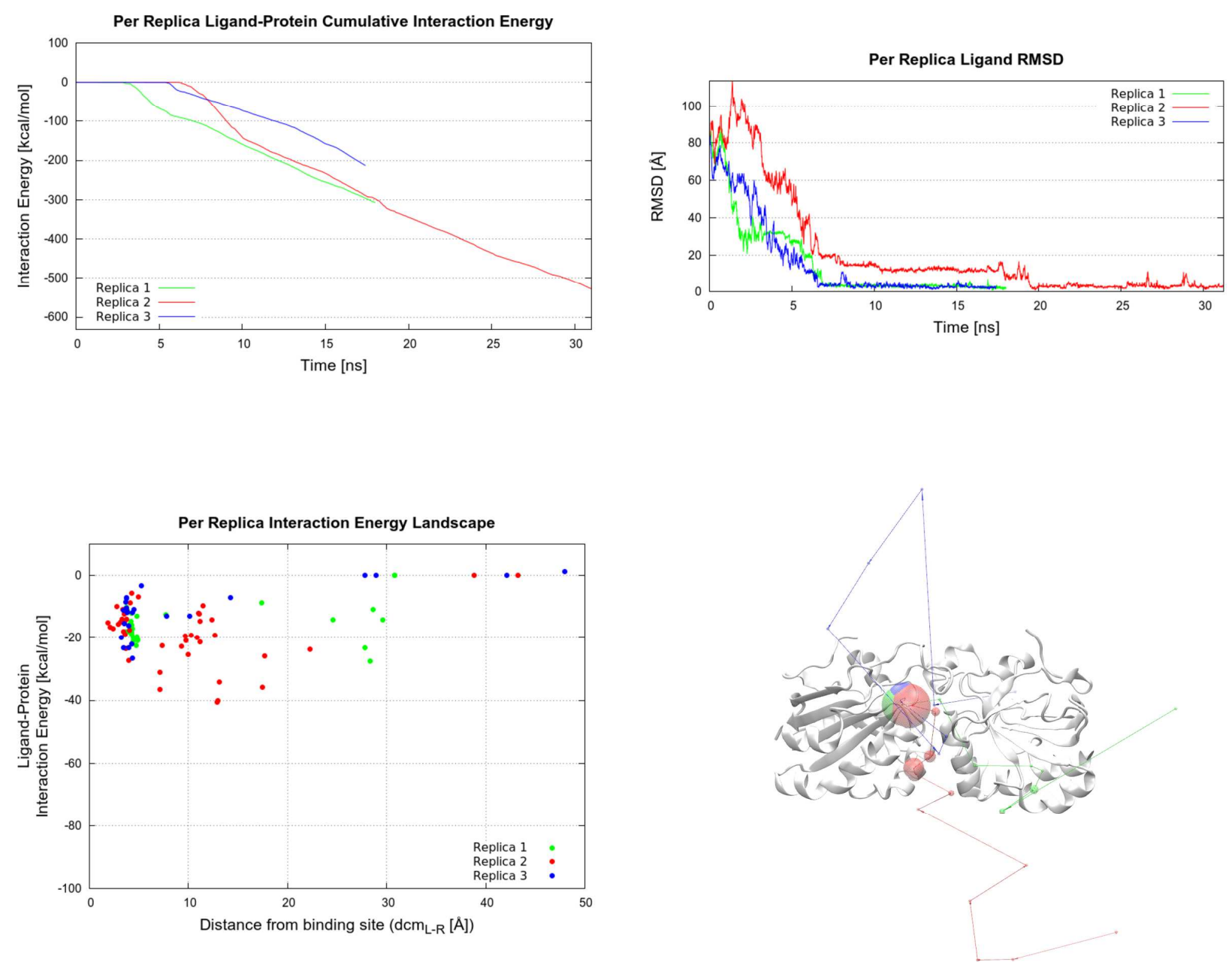
Figure S6. (S)-naproxen-HAS Supplementary analysis.

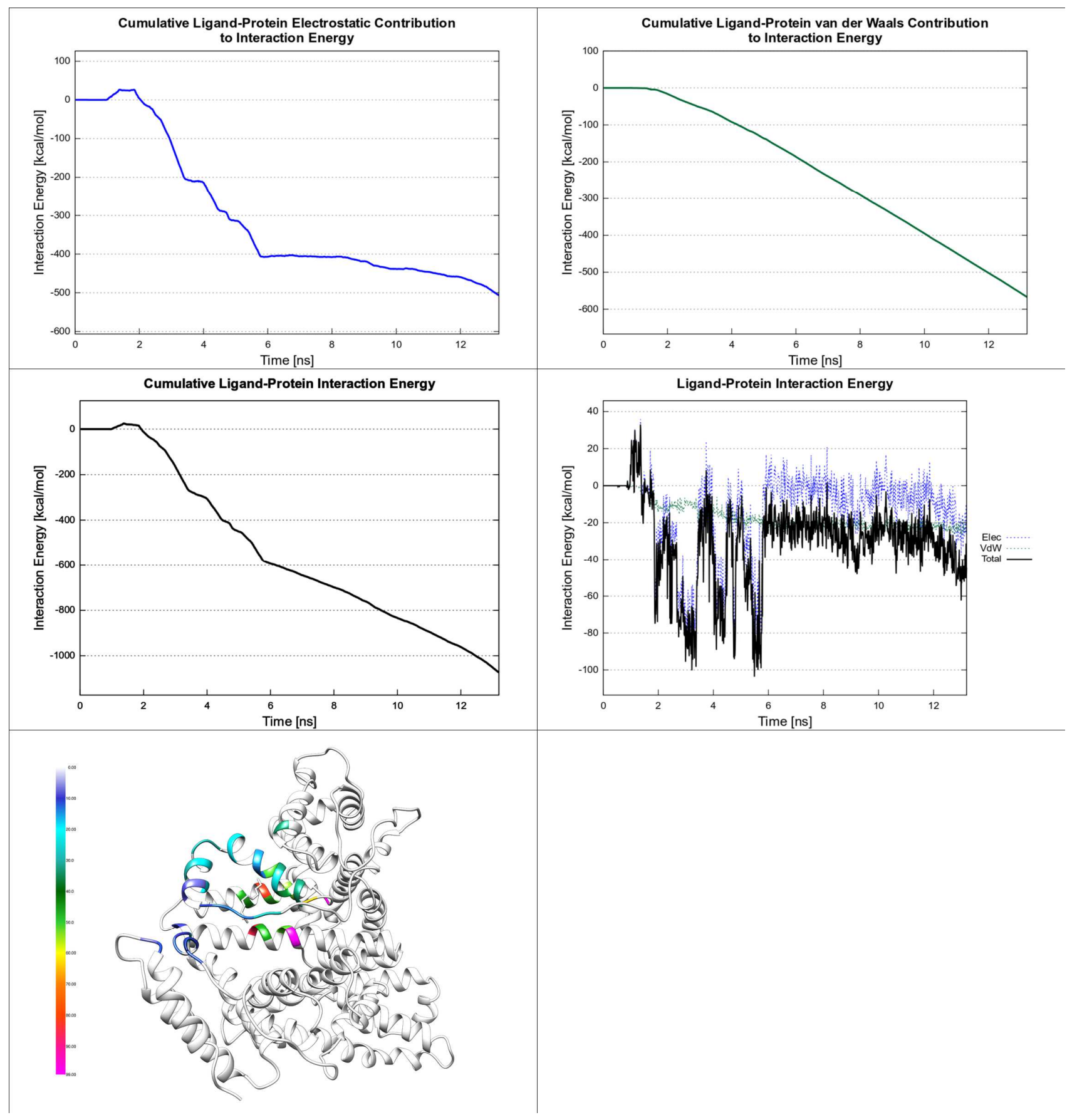


Figure S7. (S)-naproxen-HAS Replicas Analysis.
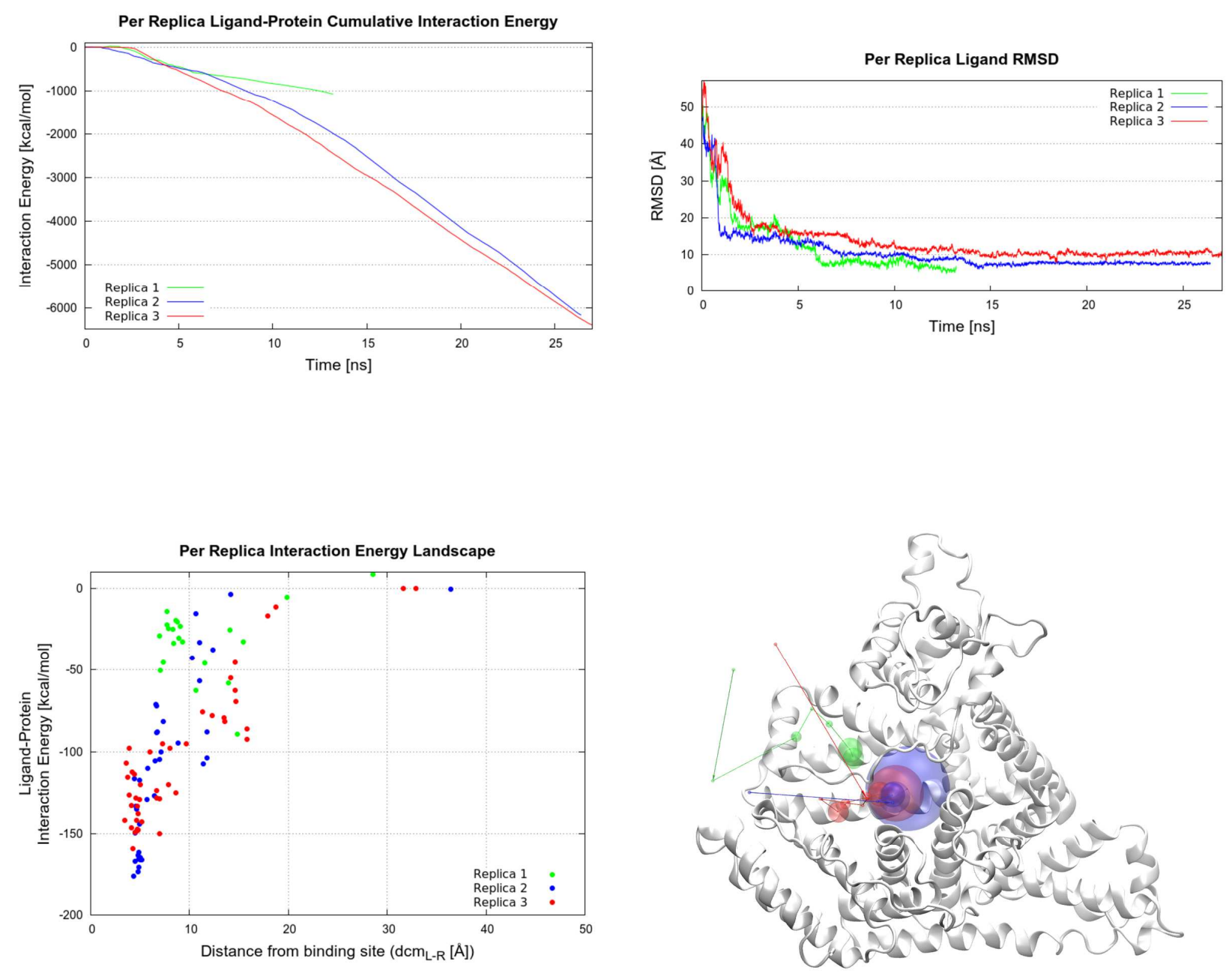
Figure S8. (S)-fluoxetine-LeuT Supplementary analysis.

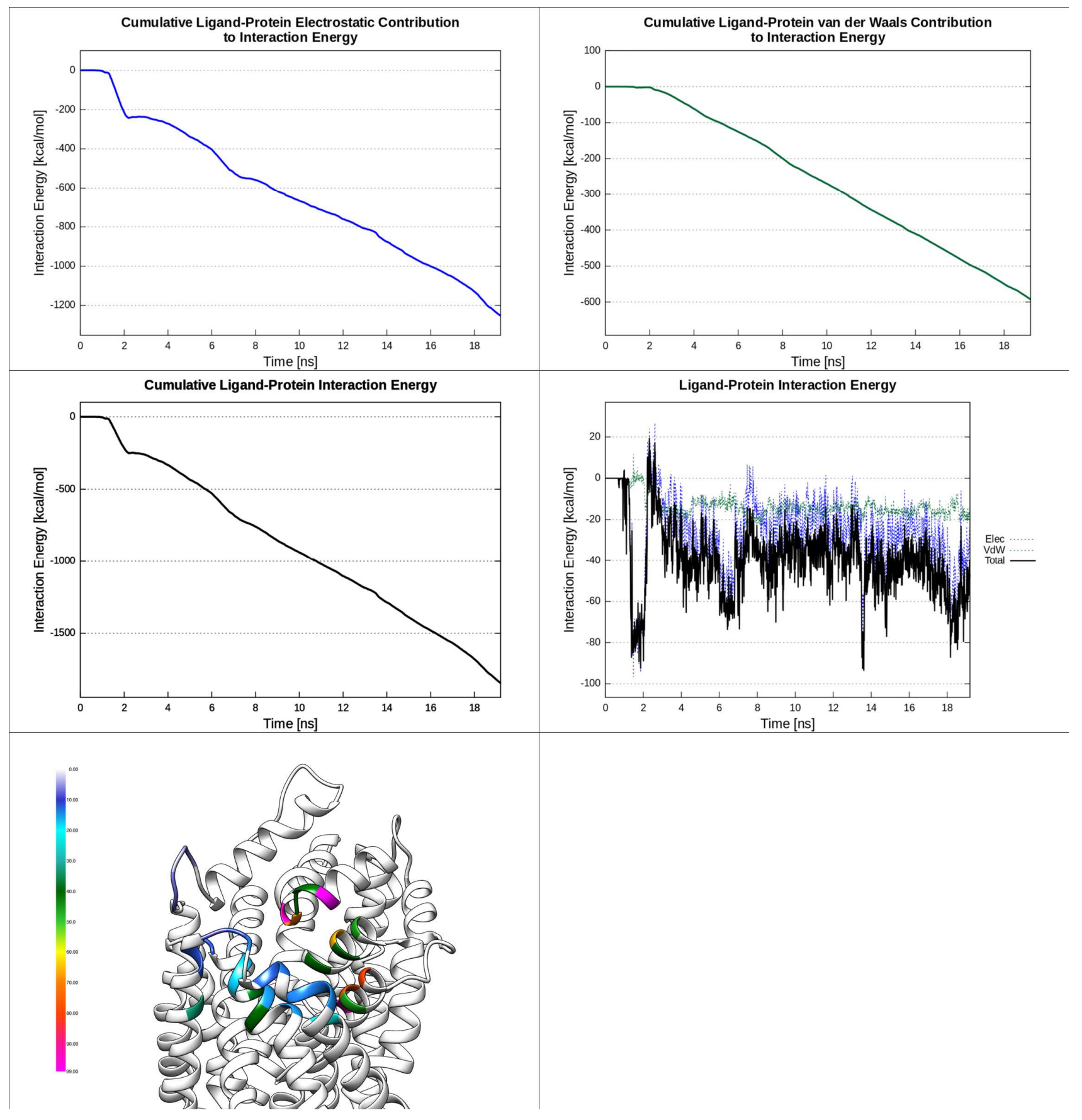


Figure S9. (S)-fluoxetine-LeuT Replicas Analysis.
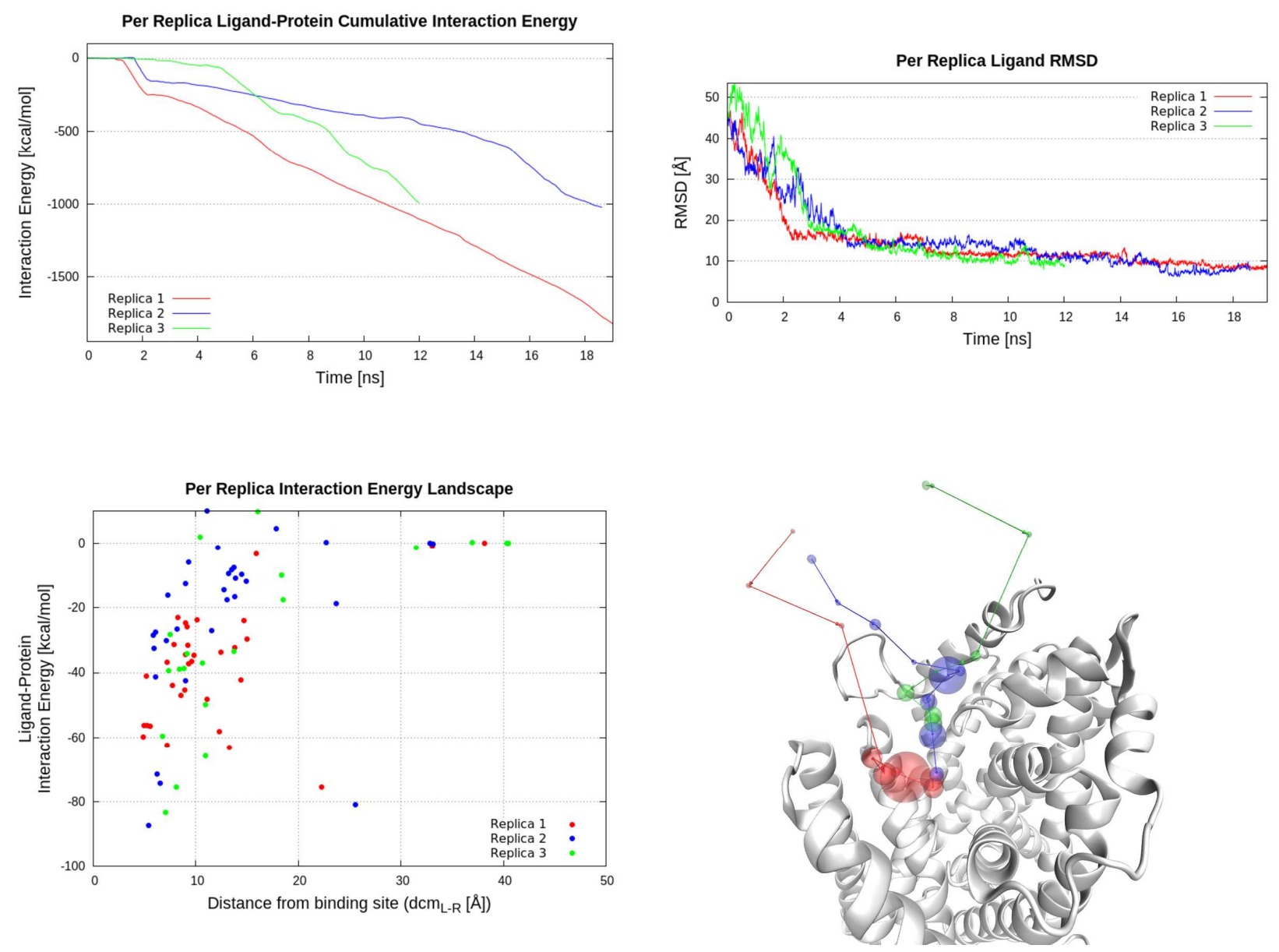
Figure S10. NECA-hA $2 \mathrm{~A}$ AR Supplementary analysis.

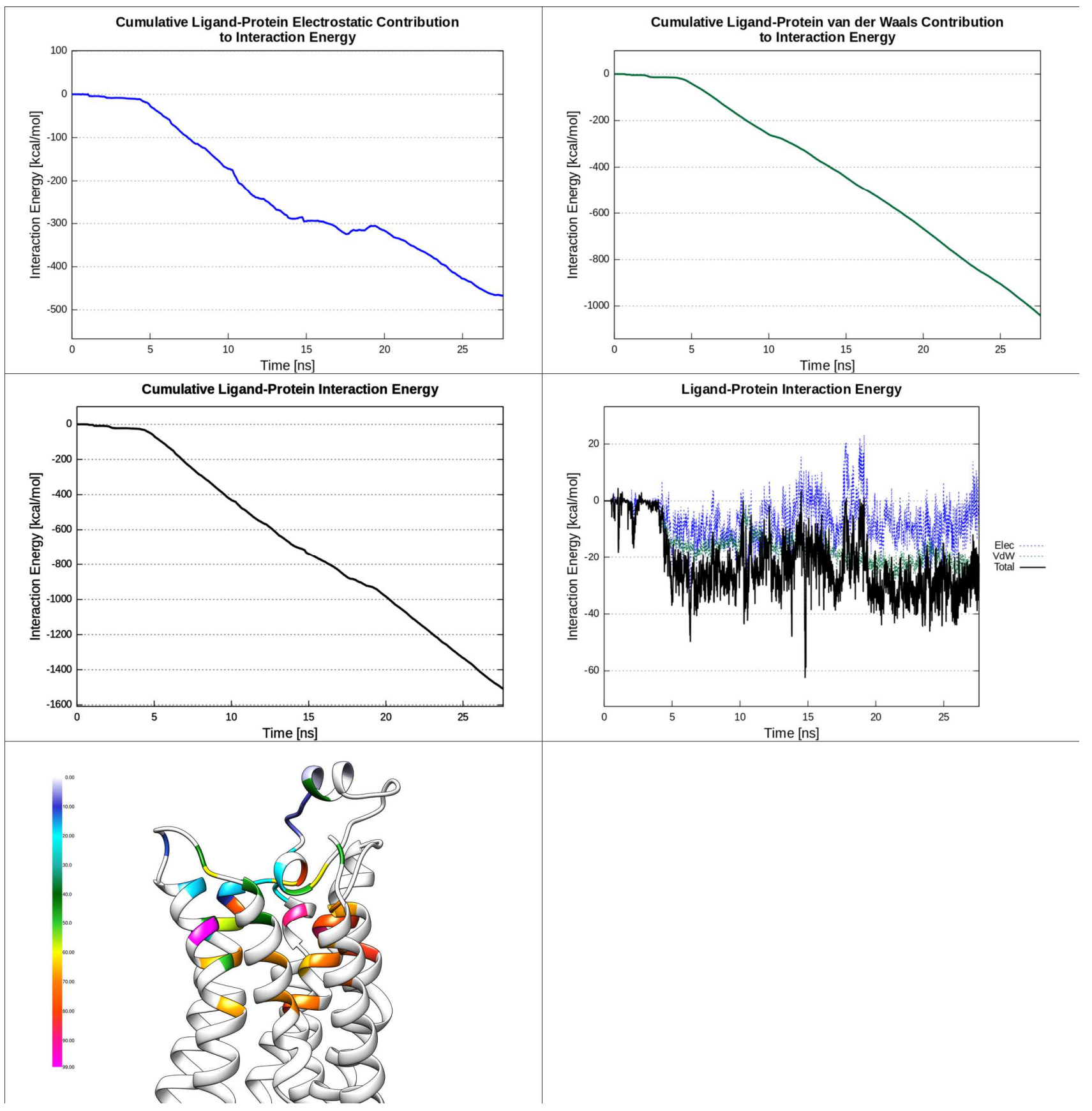


Figure S11. NECA-hA ${ }_{2 A}$ AR Replicas Analysis.
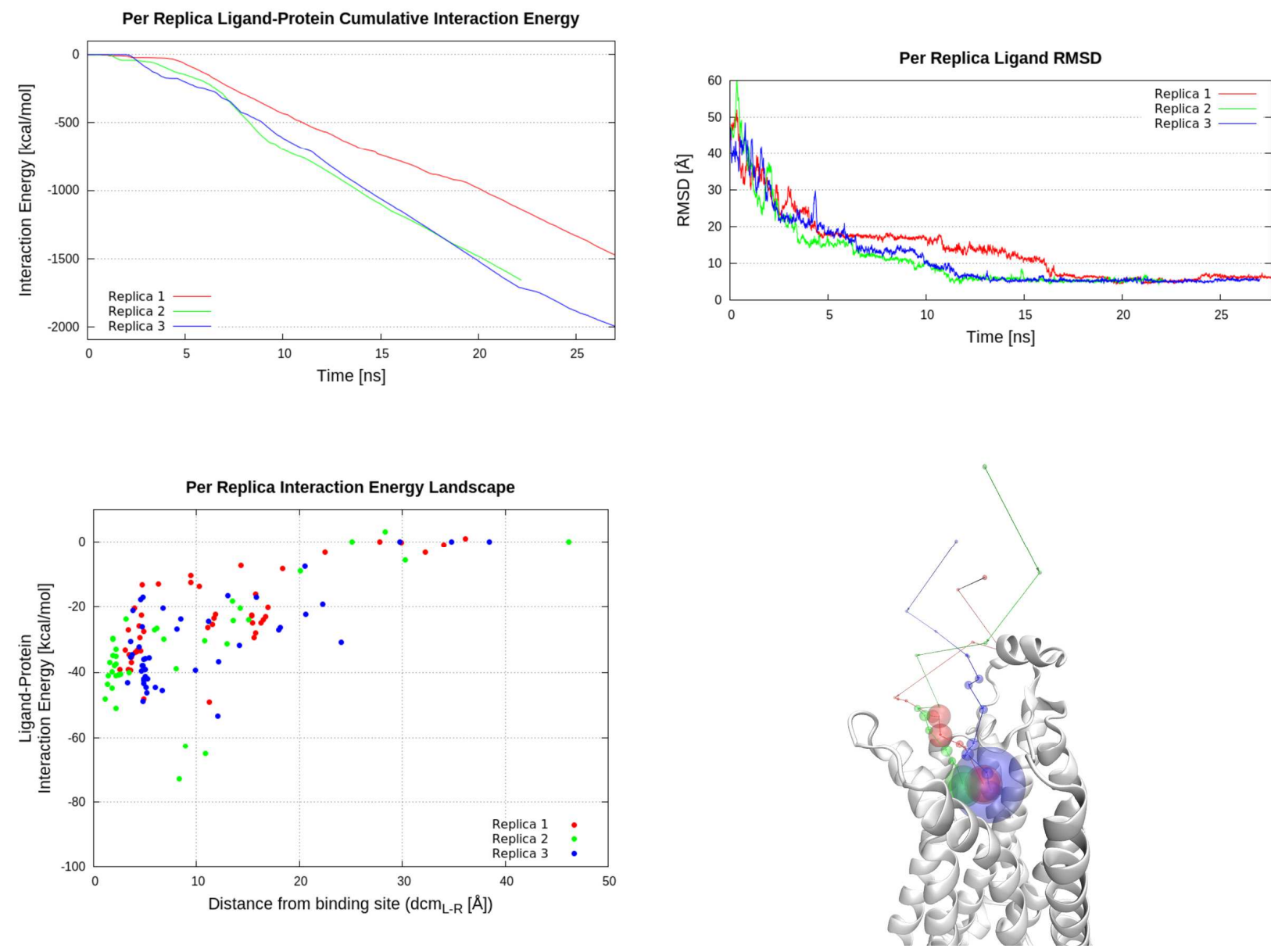
Figure S12. Alternative Arg30 conformations in LeuT X-ray crystallographic complexs. Superposition of two different LeuT X-ray crystallographic structures (PDB code 3GWW in green and PDB code 3TU0 in gray): the ionic lock situated at the extracellular side of the transporter, in absence of inhibitor, has been crystallized in a wider conformation compared to the compact topology characterizing the complex with $(S)$-fluoxetine.

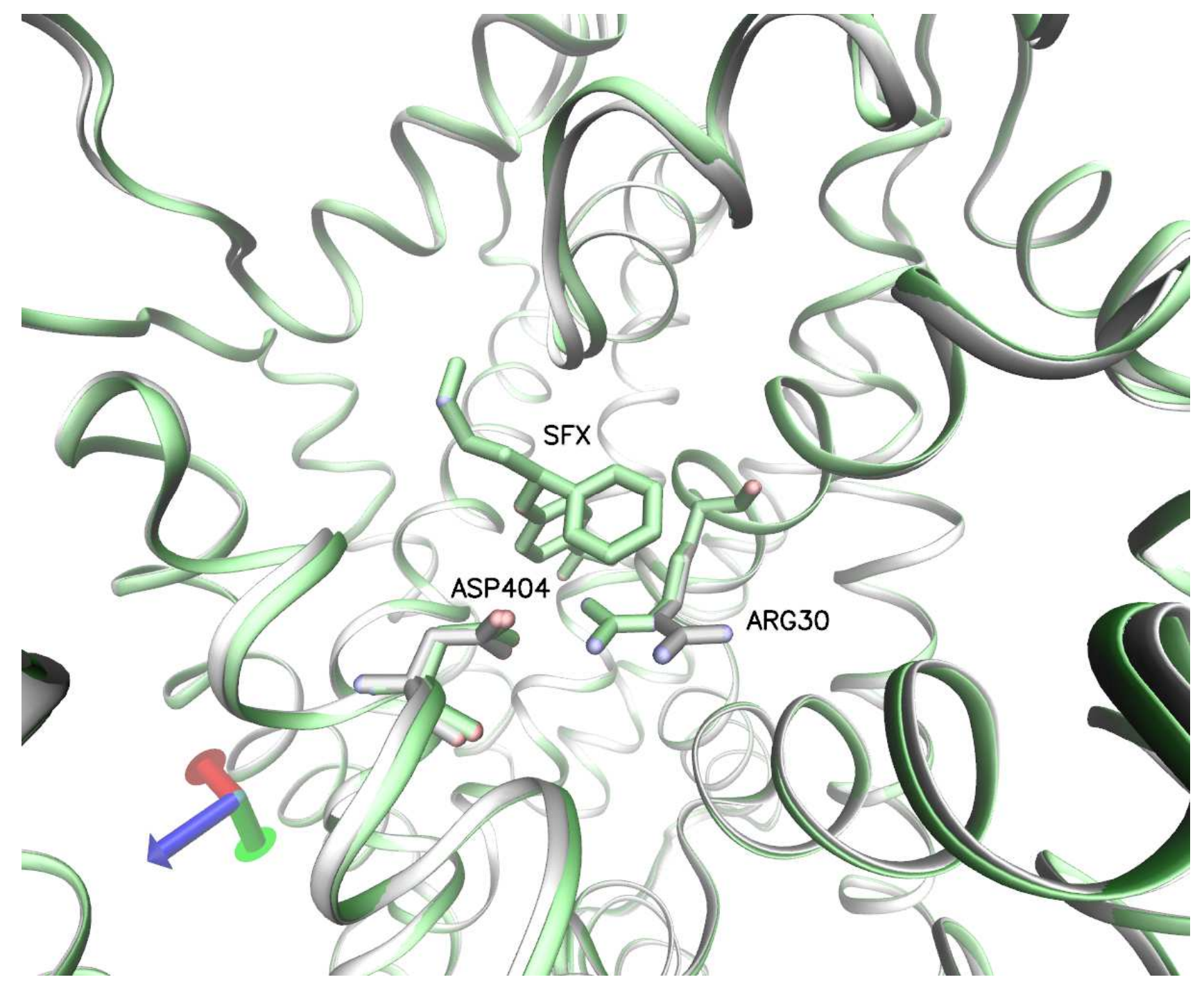

